Human and Animal Health

Vol.59: e16150131, January-December 2016 http://dx.doi.org/10.1590/1678-4324-2016150131 ISSN 1678-4324 Online Edition

BRAZILIAN ARCHIVES OF BIOLOGY AND TECHNOLOGY

AN INTERNATIONAL JOURNAL

\title{
Transgenic Expression and Identification of Recombinant Human Proinsulin in Peanut
}

\author{
Zheng Ling 1,2,3, Jiao Qi-Qing ${ }^{4}$, Wang Yu ${ }^{1,2,3}$, Bian Fei ${ }^{2,3}$, Qu Shu-Jie ${ }^{5}$, Wan Shu-Bo ${ }^{1,2,3,6}$, \\ Peng Zhen-Ying 1,2,3,Bi Yu-Ping 1,3,6*. \\ ${ }^{1}$ School of Life Science, Shandong University, Jinan, P. R. China. ${ }^{2}$ Biotechnology Research Center, Shandong \\ Academy of Agricultural Science, Jinan, P. R. China. ${ }^{3}$ Shandong Provincial Key Laboratory of Genetic \\ Improvement, Ecology and Physiology of Crops, Jinan, P. R. China. ${ }^{4}$ Shandong Institute of Pomology, Taian, P. R. \\ China. ${ }^{5}$ Test Base Service Center, Shandong Academy of Agricultural Sciences, Jinan, P. R. China. ${ }^{6}$ Center of \\ Graduate Education, Shandong Academy of Agricultural Science, Jinan, P. R. China.
}

\begin{abstract}
The increased incidence of diabetes, coupled with the introduction of alternative insulin delivery methods that rely on higher doses, is expected to result in a substantial escalation in the future demand for affordable insulin. Plant-based systems offer a safe and economical method for producing pharmaceutical proteins. We used peanut (Arachis hypogaea L.) as bio-reactors to express biosafe, stable proinsulin. We designed two proinsulin analogues (FAIA and LAIA) with substitutions in their amino acid sequences. The fast-acting insulin analogue (FAIA) contains a Gly inserted between Cys19 and Gly20, as well as a Pro28Asp substitution, in the B chain. The long-acting insulin analogue (LAIA) contains a Gly inserted between Cys 19 and Gly20 and two Arg residues inserted into the terminus of the B chain, as well as an Asn21Gly substitution in the A chain. Four plasmids were constructed: pROKII-FlagFAIA, pROKII-Flag-LAIA, pCAMBIA2301-Oleosin-FAIA and pCAMBIA2301-Oleosin-LAIA. These plasmids were transferred into peanut to produce recombinant proinsulin. Western blot and GUS staining analysis indicated that some transgenic peanut successfully expressed exogenous proinsulin. Peanut seeds can act as insulin storage sites, which is the foundation for further production of recombinant proinsulin from peanut seeds.
\end{abstract}

Key words: Proinsulin, peanut, diabetes, expression

*Authors for correspondence: yuping_bi@hotmail.com 


\section{INTRODUCTION}

In recent years, the global incidence of diabetes has increased dramatically due to genetic variations, improper diet and lifestyle (Baeshen et al. 2014). The number of diabetic patients is estimated to reach 10 percent of the world population, skyrocketing to 592 million by 2035 , with approximately 5.1 million deaths annually. Along with the skyrocketing patient numbers, the demand for insulin is increasing rapidly (more than 16 tons per year), with current insulin manufacturing technologies and production capacity unable to meet the growing market demand for high production in the future (Colagiuri 2010; Baeshen et al. 2014).

Early on, insulin was purified from the bovine or porcine pancreas. At present, E. coli and Saccharomyces cerevisiae are the most common hosts for the production of human insulin (Thim et al. 1986; Nilsson et al. 1996; Gellissen and Hollenberg 1997; Kjeldsen 2000; Porro et al. 2005; Huang et al. 2012; Ferrer-Miralles and Villaverde 2013; Baeshen et al. 2014). The large-scale manufacture of therapeutic insulin for humans has benefited tremendously from genetic engineering (Arakawa et al. 1998; Walsh 2005; Nykiforuk et al. 2006; FerrerMiralles et al. 2009; Boyhan and Daniell 2011; Qian et al. 2011). From 2004 to 2013, biopharmaceuticals were largely derived from E. Coli (24\%), yeast (13\%), mammalian cells (56\%), transgenic animals and plant expression systems $(3 \%)$ and insect cells (4\%) (Gurramkonda et al. 2010; Qian et al. 2011; Walsh 2012; Nielsen 2013; Walsh 2013; Baeshen et al. 2014).

Transgenic plant expression systems have attracted attention due to advantages such as high-capacity production, safety, inexpensive investment, and fast and easy scale-up (Arakawa et al. 1998; Nykiforuk et al. 2006; Ruhlman et al. 2007; Xie et al. 2008; Boothe et al. 2010; Boyhan and Daniell 2011; Soltanmohammadi et al. 2014). Transgenic seeds and leaves exhibit long-term stability and can be used to stockpile insulin until it is needed. Nykiforuk et al. expressed an insulin-oleosin fusion in Arabidopsis thaliana seeds, and the insulin that accumulated in the transgenic seeds could significantly reduce glucose levels to a similar extent as commercially available insulin (Nykiforuk et al. 2006). Ruhlman et al. expressed a recombinant cholera toxin Bproinsulin fusion protein (CTB-Pins) in lettuce and tobacco chloroplasts, in which CTB-Pins accounted for almost $16 \%$ and $2.5 \%$ of the total soluble protein, respectively (Ruhlman et al. 2007; Boyhan and Daniell 2011). Interestingly, the transformed tobacco leaves were able to lower blood and urine glucose levels when administered orally to non-obese diabetic mice (Ruhlman et al. 2007; Boyhan and Daniell 2011).

The preferred form of recombinant human insulin for heterologous expression is proinsulin, which has a BC-A structure and possesses a longer in vivo half-life than mature insulin (Boyhan and Daniell 2011; Trabucchi et al. 2012; Aslam et al. 2013; Soltanmohammadi et al. 2014). To change the duration of insulin's effects, fast-acting insulin analogues (FAIA), medium-acting insulin analogues (MAIA) and long-acting insulin analogues (LAIA), such as Detemir, Glargine, Ultratard, Lispro and Glulisine, have been developed. These insulin analogues were obtained by changing several amino acids in the A-Chain and/or B-Chain (Martina et al. 1989; McKeage and Goa 2001; Chen and Yao 2002; Walsh 2005; Peterson 2006), for example, Lispro and Glargine.

Peanut is an important oilseed crop worldwide. China is the biggest peanut producer, accounting for approximately $41.5 \%$ of overall global production (http://en.wikipedia.org/wiki/Peanut). We have studied peanuts for many years and have made significant advances (Su et al. 2011; Peng et al. 2013; Xia et al. 2013). Peanut seeds are rich in protein and fat and can serve as a good reservoir for heterologously expressed proteins; thus, we sought to express the human insulin gene in peanut seeds. Because insulin is highly unstable and is prone to $\mathrm{N}$ terminal degradation (Boothe et al. 2010), oleosin or flag tag was fused with proinsulin to protect the Nterminus from degradation. Otherwise the recombination protein purification cost will be reduced. Here, we share our experimental results with researchers in this field.

\section{MATERIAL AND METHODS}

\section{Plasmid Design and Construction}

In accordance with previous reports (Martina et al. 1989; McKeage and Goa 2001; Chen and Yao 2002; Walsh 2005; Nykiforuk et al. 2006; Peterson 2006), we designed two types of proinsulin analogues: a FAIA and a LAIA (Fig. 1). In the FAIA, Gly was inserted between Cys19 and Gly20, and Pro28 was replaced with Asp28, in the B chain. In the LAIA, Gly was inserted between Cys19 and Gly20 and two 
Arg residues were inserted at the $\mathrm{C}$-terminus of the $\mathrm{B}$ chain, and Asn21 was replaced with Gly21 in the A chain. We optimized the codon according to the plant condon preference. The two human proinsulin analogues were synthesized by Sangon Company (China). An eight-residue flag tag was fused at the Nterminus of the FAIA and LAIA. Flag-LAIA and Flag-FAIA fragments were amplified using the primers LAIAF and LAIAR or FAIAF and FAIAR and inserted downstream of the CaMV35S promoter in the binary expression vector pROKII, which carries the marker gene $\mathrm{Npt}$ II. The resulting plasmids were named pROKII-Flag-FAIA and pROKII-FlagLAIA (Fig. 2).
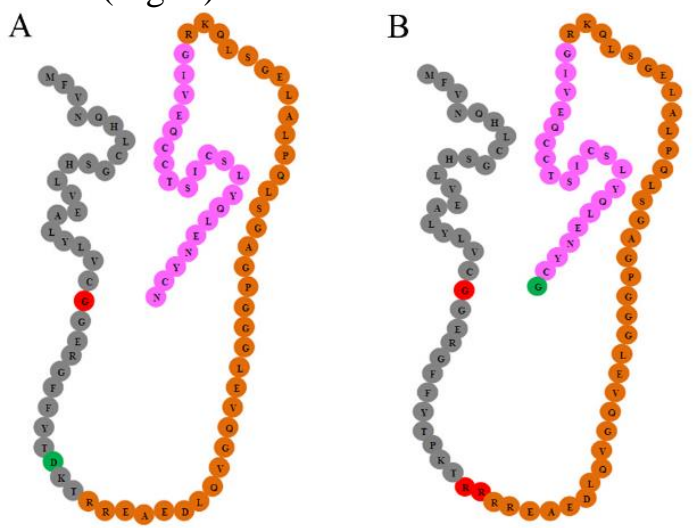

Figure. 1 Amino acid sequences of FAIA and LAIA. A: FAIA; B: LAIA. Grey circles represent the B-chain, orange circles represent the C-peptide, pink circles represent the A-chain, red circles represent the inserted amino acids, and green circles represent the substituted amino acids.
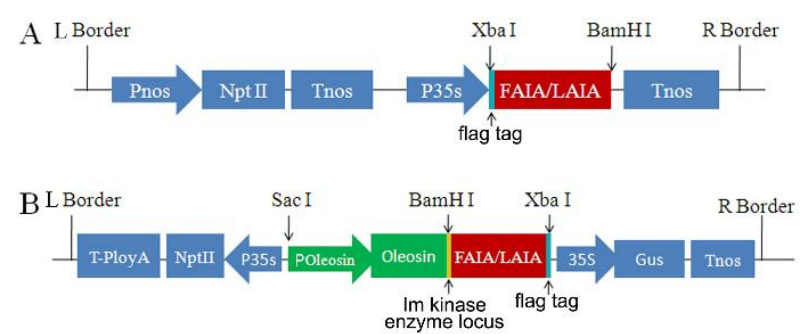

Figure. 2 The schematic diagram of the recombinant plasmids. A: pROKII-Flag-FAIA/LAIA plasmids; B: pCAMBIA2301-Oleosin-FAIA/LAIA plasmids.

Total RNA was extracted from young seeds of the peanut cultivar 'Luhua 14' using the CTAB method (Albani et al. 1992) and transcribed to cDNA using a RevertAid First Strand cDNA Synthesis kit (Fermentas, Canada). The primers OLEOSINF and OLEOSINR (Table S1) were used to amplify the peanut oleosin gene fragment and its promoter (GenBank No. EF695400). PCR amplification was conducted as follows: one cycle of $95^{\circ} \mathrm{C}$ for $5 \mathrm{~min}$, 35 cycles of $94^{\circ} \mathrm{C}$ for $30 \mathrm{~s}, 57^{\circ} \mathrm{C}$ for $30 \mathrm{~s}$, and $72^{\circ} \mathrm{C}$ for $2 \mathrm{~min}$, and a final extension at $72^{\circ} \mathrm{C}$ for $5 \mathrm{~min}$.

Table.S1 - Primers used in this paper

\begin{tabular}{lll}
\hline Primer & Sequence & Function \\
\hline LAIAF & 5'GCCTCTAGAATGGATTACAAGGATG & Amplify and detect the LAIA fragment in \\
& ATGA 3' $($ Xba I $)$ & pROKII-Flag-LAIA vector \\
LAIAR & 5'GCCGGATCCTTACCCGCAATAATTC & \\
& TCG 3' (BamH I) & \\
FAIAF & 5'GCCTCTAGAATGGACTATAAAGATG & Amplify and detect the FAIA fragment in \\
& ATGA 3' (Xba I) & pROKII-Flag-LAIA vector \\
FAIAR & 5'GCCGGATCCTTAATTGCAGTAGTTT & \\
& TCAA3' (Bam H I) & \\
OLEOSINF & 5'GCCGAGCTCAGGTCAACTACCATTC & Amplify the peanut oleosin gene fragment and \\
& GT 3' (Sac I $)$ & its promoter \\
\hline
\end{tabular}




\begin{tabular}{|c|c|c|}
\hline \multirow[t]{2}{*}{ OLEOSINR } & \multicolumn{2}{|l|}{ 5'GCCGGATCCAGTTCTCTTTGAATCC } \\
\hline & TG 3’ (BamH I) & \\
\hline \multirow[t]{3}{*}{ O-LAIAF } & 5'GCCGGATCCGATGATGATGATAAG & Amplify the O-FAIA fragment in \\
\hline & ATGTTTGTAAACCAGCACCTC 3' & pCAMBIA2301-Oleosin-LAIA vector \\
\hline & $($ BamH I) & construction \\
\hline \multirow[t]{3}{*}{ O-LAIAR } & 5'GCCTCTAGATTACTTATCGTCATCA & \\
\hline & TCCTTGTAATCCCCGCAATAATTCTCG & \\
\hline & AGTT 3' (Xba I) & \\
\hline \multirow[t]{3}{*}{ O-FAIAF } & 5'GCCGGATCCGATGATGATGATAAG & Amplify the O-LAIA fragment in \\
\hline & ATGTTTGTGAACCAGCACCTC 3' & pCAMBIA2301-Oleosin vector construction \\
\hline & $($ BamH I) & \\
\hline \multirow[t]{3}{*}{ O-FAIAR } & 5'GCCTCTAGATTACTTATCGTCATCA & \\
\hline & TCTTTATAGTCATTGCAGTAGTTTTCA & \\
\hline & AGTTG 3' (Xba I) & \\
\hline \multirow[t]{2}{*}{ RTR } & 5'GCCCGCAAACCGGAAAACAAACGT & Detect the pCAMBIA2301-Oleosin- \\
\hline & TAAGT 3' & FAIA/LAIA transgenic lines \\
\hline ACTINF & 5'GGAACTGGAATGGTTAAGGCTGG 3' & Amplify the $\beta$-actin gene in RT-PCR \\
\hline ACTINR & 5'GTTTCAAACATGATCTGAGTCATC 3' & \\
\hline
\end{tabular}

The PCR products were separated by $1.0 \%$ agarose gel electrophoresis. O-LAIA and O-FAIA were amplified using the primer pairs O-LAIAF/OLAIAR and O-FAIAF/O-FAIAR. In addition, an eight-residue flag tag was added to the C-terminus of O-FAIA and O-LAIA. The oleosin promoter and gene fragment were fused to the N-terminus of $\mathrm{O}$ FAIA and O-LAIA via an enterokinase enzyme site. The fused O-FAIA and O-LAIA fragments were inserted into the binary expression vector pCAMBIA2301, which carries the marker gene $N p t$ II. The resulting plasmids were named pCAMBIA2301-Oleosin-FAIA and pCAMBIA2301-Oleosin-LAIA (Fig. 2).

The four recombinant plasmids were transferred into Agrobacterium tumefaciens strain LBA4404
(Hoekema et al. 1983) for the genetic transformation of peanut.

\section{Genetic Transformation of Peanut Axes}

Mature embryo axes of peanuts were used to induce explants as described previously (Akasaka et al. 2000; Anuradha et al. 2006). Briefly, The axes were cut from the young seedlings (about 4-5 days old) of 'Luhua 14' (a Chinese peanut cultivar) and mixed with the transformed A. tumefaciens strain LBA4404 bacterial suspension for about $25 \mathrm{~min}$, then put the axes on coincubate-medium (MS added $8 \mathrm{mg} / \mathrm{L} 6-\mathrm{BA}$ and $0.7 \mathrm{mg} / \mathrm{L}$ NAA) under dark conditions for two days. The infected axes were transferred to inductionmedium (MS added $50 \mathrm{mg} / \mathrm{L}$ Kan, $250 \mathrm{mg} / \mathrm{L}$ Cef, 8 $\mathrm{mg} / \mathrm{L} 6-\mathrm{BA}$ and $0.7 \mathrm{mg} / \mathrm{L} \mathrm{NAA}$ ) for sprouting for 34 weeks. The well-elongated green shoots were cut 
off from the axes and put on the elongation-medium (MS added $100 \mathrm{mg} / \mathrm{L}$ Kan, $250 \mathrm{mg} / \mathrm{L} \mathrm{Cef,} 2 \mathrm{mg} / \mathrm{L} 6$ BA and $2.0 \mathrm{mg} / \mathrm{L} \mathrm{GA3}$ ) for 3-4 weeks, then transfer the green shoots on rooting-medium (MS added 0.5 $\mathrm{mg} / \mathrm{L}$ IBA). About 3-4 weeks later, 5-8 strong roots will developed from the stem bases. The well-rooted plants were cultivated in pots in a greenhouse for production. The well-rooted plants were planted in pots with soil and cultivated in a controlled chamber at $28^{\circ} \mathrm{C}$ with a relative humidity of $50-60 \%$.

The seeds from the positive transgenic peanut lines were grown in greenhouse (i.e., the T1 generation). All of the plants were identified using PCR. Positive plants from transgenic lines were kept for seed harvest. Such seeds (T2, T3 generation) were planted in greenhouse and identified. These T3 lines without character segregation were chosen for western blot analysis.

\section{Molecular Identification of Transgenic and Peanut Plants}

Genomic DNA was extracted from the young leaves of T0, T1 and T2 transgenic peanut lines (Wang et al. 2002). Four pairs of primers were used for the identification of all the transgenic plants. Primers LAIAF/LAIAR and FAIAF/FAIAR were used to amplify the Flag-LAIA and Flag-FAIA fragments. Primers O-LAIAF/RTR and O-FAIAF/RTR were used to amplify the O-LAIA/O-FAIA fragments. PCR amplification was conducted as follows: one cycle of $95^{\circ} \mathrm{C}$ for $5 \mathrm{~min}, 35$ cycles of $94^{\circ} \mathrm{C}$ for $30 \mathrm{~s}$, $52^{\circ} \mathrm{C}$ for $45 \mathrm{~s}$, and $72^{\circ} \mathrm{C}$ for $1 \mathrm{~min}$, followed by a final extension at $72^{\circ} \mathrm{C}$ for $5 \mathrm{~min}$. The PCR products were separated by $1.5 \%$ agarose gel electrophoresis.

\section{Histochemical Assay for GUS Activity}

Both WT and positive transgenic peanut cotyledons were histochemically stained to test for GUS activity according to the method of Jefferson et al (Jefferson et al. 1987).

\section{RT-PCR Analysis}

Total RNA was extracted using the CTAB method from the leaves of T2 transgenic peanut lines. The two primer pairs LAIAF/LAIAR and FAIAF/FAIAR were used for RT-PCR analysis. $\beta$-actin was amplified as a reference gene using the primer pair ACTINF/ACTINR. PCR was conducted as follows: one cycle of $95^{\circ} \mathrm{C}$ for $5 \mathrm{~min}, 22$ cycles of $94^{\circ} \mathrm{C}$ for 30 $\mathrm{s}, 55^{\circ} \mathrm{C}$ for $30 \mathrm{~s}$, and $72^{\circ} \mathrm{C}$ for $1 \mathrm{~min}$, followed by a final extension at $72^{\circ} \mathrm{C}$ for $5 \mathrm{~min}$. The PCR products were separated by $1.5 \%$ agarose gel electrophoresis.

\section{Extraction of Protein from Transgenic Peanut}

Total proteins were extracted from pROKII-FlagFAIA/LAIA T2 transgenic peanut leaves using the trichloroacetic acid (TCA)/acetone method. Plant leaves were crushed in liquid nitrogen, and 10\% TCA acetone (supplied with $0.07 \%$ 2-mercaptoethanol and $1 \mathrm{mM}$ PMSF) was added. The mixture was placed at $-20^{\circ} \mathrm{C}$ for $1 \mathrm{~h}$ and microfuged at $12,000 \mathrm{rpm}$ for 30 min in $4^{\circ} \mathrm{C}$. The supernatant was discarded. Precooled acetone was added to the precipitate, which was placed at $-20^{\circ} \mathrm{C}$ for $1 \mathrm{~h}$ and then microfuged at $12,000 \mathrm{rpm}$ for $30 \mathrm{~min}$ at $4^{\circ} \mathrm{C}$. This procedure was repeated twice. The final precipitate was vacuum dried, resuspended in $400 \mu 1$ lysis buffer (urea $7 \mathrm{M}$, thiourea $2 \mathrm{M}$, CHAPS $4 \%$, DTT $65 \mathrm{mM}$, PMSF $1 \mathrm{mM}$ ), incubated for $20 \mathrm{~min}$ at room temperature, and then microfuged at 12,000 rpm for $30 \mathrm{~min}$ at $15^{\circ} \mathrm{C}$. The supernatant contained the total protein extract.

Oleosin-proinsulin was extracted from $\mathrm{T} 2$ transgenic plants seeds using the method of Markley and Nykiforuk (Markley et al. 2006; Nykiforuk et al. 2006).

\section{Western Blot Analysis}

Protein content was determined using the bicinchoninic acid (BCA) protein assay kit (Pierce, Rockford, IL, USA) according to the manufacturer's instructions. A $40 \mu 1$ sample was taken and added to $10 \mu \mathrm{l}$ of sodium dodecyl sulfate (SDS) buffer (250 $\mathrm{mM}$ Tris- $\mathrm{HCl}, \mathrm{pH} 6.8,10 \%$ SDS, $0.5 \%$ bromophenol blue, $50 \%$ glycerol, 5\% 2-mercaptoethanol) and boiled for $10 \mathrm{~min}$. Western blot analysis was operated according the method described previously (Nykiforuk et al. 2006). The $0.22-\mu \mathrm{m}$ polyvinylidene difluoride membranes, monoclonal anti-insulin and alkaline phosphatase-conjugated goat anti-rabbit IgG were bought from AMRESCO (Canada), Abcam (America) and ZSGB-BIO (China).

\section{RESUILTS}

\section{Molecular Identification of Transgenic Plants}

We designed two types of proinsulin analogues: a FAIA and a LAIA (Fig. 1), and constructed pROKIIFlag-FAIA/LAIA and pCAMBIA2301-OleosinFAIA/LAIA. The four plasmids were transformed into peanut mature embryo axes using Agrobacterium-mediated transformation. There were no phenotype difference between wide type and 
transgenic lines. Finally several transgenic peanut lines were obtained (Fig. S1). The numbers of transgenic plants lines were in Table 1.

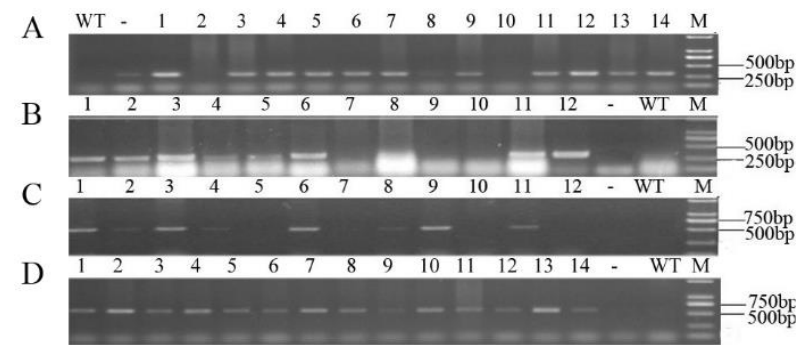

Table S1 Primers used in this study

Figure S1. PCR tests of transgenic peanut lines. A: pROKII-Flag-FAIA transgenic peanut lines; B: pROKIIFlag-LAIA transgenic peanut lines; C: pCAMBIA2301Oleosin-FAIA transgenic peanut lines; D: pCAMBIA2301-Oleosin-LAIA transgenic peanut lines. M: DNA marker, -: negative control, WT: wild type 'Luhua14'.

Table 1 - The number of transgenic peanut lines

\begin{tabular}{ll}
\hline Plasmid & Transgenic peanut (lines) \\
\hline pROKII-Flag-FAIA & 20 \\
pROKII-Flag- LAIA & 7 \\
pCAMBIA2301-Oleosin-FAIA & 21 \\
pCAMBIA2301-Oleosin- LAIA & 14 \\
\hline
\end{tabular}

T2 transgenic peanut lines were randomly chosen for RT-PCR analysis to examine LAIA and FAIA expression in the peanut. Total mRNA was extracted from young leaves of both WT and positive lines. Most of the transgenic peanut lines expressed high levels of LAIA or FAIA mRNA. LAIA-7 and FAIA3 expressed highest proinsulin levels, but lines LAIA-3 and FAIA-5 which expressed little or no proinsulin (Fig. 3).

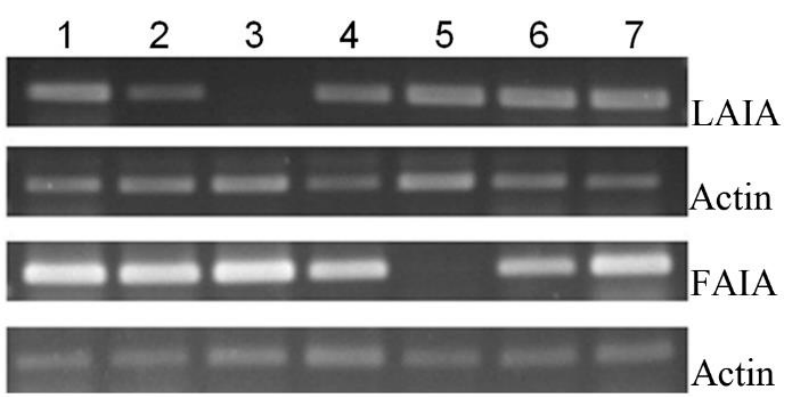

Figure. 3 RT-PCR analysis of T2 transgenic peanut lines. 1-7: T2 transgenic lines.

Because the pCAMBIA2301-Oleosin-FAIA/LAIA plasmids contain the GUS gene, we performed GUS staining to further identify the positive transgenic peanut lines. All the cotyledons of the positive transgenic peanut were deeply stained and the WT showed no staining (Fig. 4), thus demonstrating the successful transformation of the peanut plants.
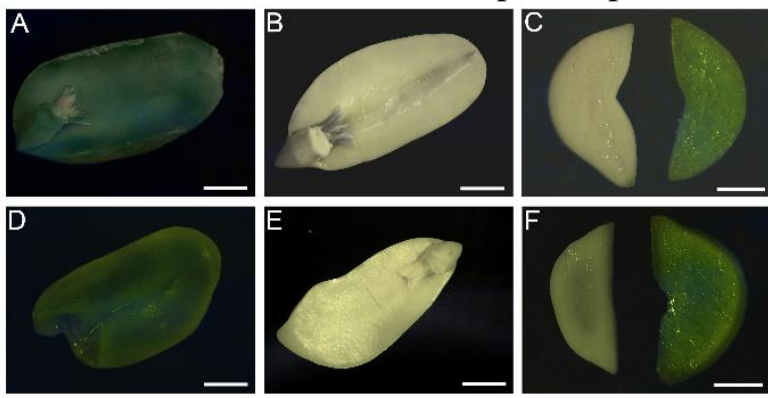

Figure. 4 GUS staining assay. A-C: pCAMBIA2301Oleosin-FAIA transgenic peanut lines; D-F: pCAMBIA2301-Oleosin-LAIA transgenic peanut lines. A, D: GUS expression in all of the cotyledons. B, E: cotyledons of WT peanut without GUS activity. C, F: transverse sections of $\mathrm{A}, \mathrm{B}, \mathrm{D}$ and $\mathrm{E}$. WT: wild type 'Luhua 14', Bars: $0.2 \mathrm{~cm}$.

\section{Western Blot Analysis in Transgenic Plants}

Western blot analysis showed that some of the T2 transgenic peanut successfully expressed the exogenous proinsulin (Fig. 5). Of the pROKII-Flag- 
FAIA/LAIA transgenic peanut lines, four lines expressed either FAIA or LAIA successfully (Fig. 5 A), but most of the peanut lines had more than one bands and had different size. In pCAMBIA2301Oleosin-FAIA/LAIA transgenic peanut lines, six positive lines expressed Oleosin-FAIA/LAIA protein (Fig. 5 B).

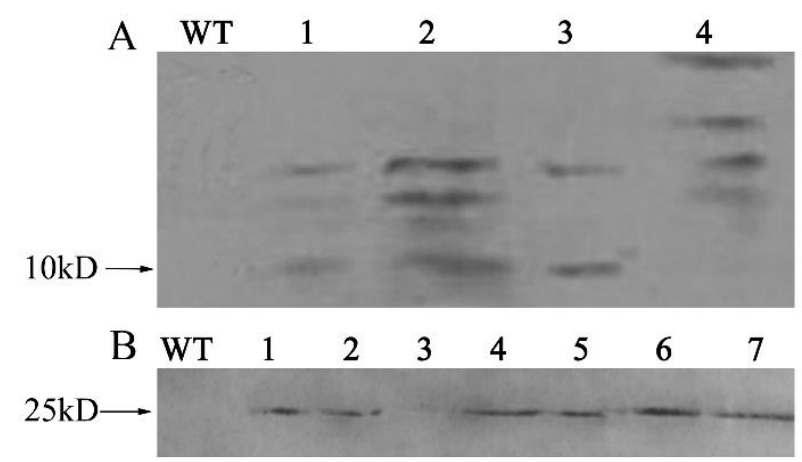

Figure. 5 Western blot analysis of $T 2$ transgenic lines. A: pROKII-Flag-FAIA/LAIA transgenic peanut line leaves. 1-2: pROKII-Flag-FAIA transgenic peanut line leaves; 3-4: pROKII-Flag-LAIA transgenic peanut line leaves. B: pCAMBIA2301-Oleosin-FAIA/LAIA transgenic peanut line seeds. 1-4: pCAMBIA2301Oleosin-FAIA transgenic peanut line seeds; 5-7: pCAMBIA2301-Oleosin-LAIA transgenic peanut line seeds.

\section{DISSUSSION}

Plant genetic engineering has been a very important technique for improving the economic characteristics of crops and enhancing their disease resistance. Recently, the use of plants as bioreactors to produce medical proteins such as antibodies and vaccines has emerged as a new trend. An obvious advantage is that the costs of plant-derived medical proteins are far lower than those of microorganism-derived or animal-derived medical proteins. Additionally, transgenic seeds exhibit long-term stability, enabling medical proteins to be stockpiled until they are needed.

Human insulin has been transformed into several plants, including Arabidopsis, lettuce, tobacco, and tomato (Nykiforuk et al. 2006; Ruhlman et al. 2007; Boyhan and Daniell 2011; Soltanmohammadi et al. 2014). Because insulin is highly unstable and is prone to N-terminal degradation (Boothe et al. 2010), it is often fused with a marker protein such as CTB or oleosin to protect the N-terminus from degradation (Nykiforuk et al. 2006; Boyhan and Daniell 2011). Here, we designed two strategies to produce human proinsulin in peanut, one strategy involved fusing a flag tag to the N-terminus of FAIA/LAIA, and the other involved fusing peanut oleosin to the Nterminus of FAIA/LAIA, with a flag tag linked to the C-terminus. The flag tag and oleosin protect FAIA/LAIA from degradation and provide a good means of facilitating purification, and the FlagFAIA/LAIA and Oleosin-FAIA/LAIA fusion proteins were successfully produced from peanut leaves and seeds (Fig. 5).

Insulin and proinsulin have been reported to form self-aggregates of monomers, dimers, tetramers and hexamers (Pekar and Frank 1972), which causes insulin and proinsulin to be more resistant to degradation. Boyhan et al. produced cholera toxin B -fused human proinsulin in tobacco and lettuce chloroplasts and found that the fusion protein aggregated in chloroplasts (Boyhan and Daniell 2011). In our experiments, exogenous proinsulin formed more than one bands and had different size in pROKII-Flag-FAIA/LAIA T2 transgenic peanut lines (Fig. 5 A). Maybe proinsulin aggregated and formed polymers. This may account for the high levels of FAIA/LAIA expression in the transgenic peanut leaves. In order to determine the proinsulin expression, we used ELISA (Ultra Sensitive Rat Insulin ELISA kit; Crystal Chemical Inc., Downers Grove, IL) (Nykiforuk et al. 2006) to detect pROKIIFlag-FAIA/LAIA T2 transgenic lines which were used in the western blot. The results revealed that all transgenic lines had considerably lower proinsulin expression levels (approximately $0.7 \%$ of total soluble protein) in stable transgenic plants than other researches previously. We speculate the reasons of low detection level may be that the ELISA kit we used is sensitive to insulin not proinsulin. We will further study the reason of this.

Currently, insulin products such as Detemir, Glargine, Ultratard, Lispro and Glulisine, etc., can be divided into different types based on their different rates of action. Lispro is one kind of fast-acting insulin that can act in 5-15 min after subcutaneous injection, the reason being that Lispro has two amino acid changes in the B-chain compared to native insulin. Glargine has a total of three different amino acids compared with native insulin, which extends the duration of its activity (McKeage and Goa 2001). Additionally, Chen et al. reported that inserting a Gly between B23 and B24 of the B-chain increases the activity of this insulin analogue compared with native human and pig insulin (Chen and Yao 2002) In accordance with these reports, we designed our own proinsulin analogues in the hopes that they will possess all the merits of Lispro and Glulisine, etc. 
Now that we have successfully expressed proinsulin analogues in peanut seed, proinsulin will be purified and treated with enterokinase and trypsin and test the matured insulin activity in future studies.

\section{CONCLUSIONS}

We used peanut as bio-reactors to express proinsulin. Two proinsulin analogues (FAIA and LAIA) were designed and transferred into peanut to produce recombinant proinsulin. Western blot and GUS staining analysis indicated that some transgenic peanut successfully expressed exogenous proinsulin. This research lay a foundation for further production of recombinant proinsulin from peanut seeds, and promoted the development of molecular farming.

\section{ACKNOWLEDGEMENTS}

This work was supported by the International Science \& Technology Cooperation Program of China (2012DFA30450), Natural Science Foundation of Shandong Province (ZR2013CM036), Shandong Province Germplasm Innovation and Utilization Project. The funders had no role in study design, data collection and analysis, decision to publish, or preparation of the manuscript.

\section{REFERENCES}

Akasaka YH, Daimon M, Mi M. Improved plant regeneration from cultured leaf segments in peanut (Arachis hypogaea L.) by limited exposure to thidiazuron. Plant Science. 2000; 156: 169-175.

Albani D, Sardana R, Robert LS, Altosaar I, Arnison PG, Fabijanski SF. A Brassica napus gene family which shows sequence similarity to ascorbate oxidase is expressed in developing pollen. Molecular characterization and analysis of promoter activity in transgenic tobacco plants. The Plant Journal. 1992; 2: 331-342.

Anuradha TS, Jami SK, Datla RS, Kirti PB. Genetic transformation of peanut (Arachis hypogaea L.) using cotyledonary node as explant and a promoterlessgus: nptII fusion gene based vector. J Biosci. 2006; 31: 235246.

Arakawa T, Yu JC, Daniel KX, Hough J, Engen PC, Langridge WHR. A plant-based cholera toxin B subunitinsulin fusion protein protects against the development of autoimmune diabetes. Nature Biotechnology. 1998; 16: 934-938.

Aslam F, Gardner QT, Zain H, Nadeem MS, Ali M, Rashid N, Akhtar M. Studies on the expression and processing of human proinsulin derivatives encoded by different
DNA constructs. Biochim Biophys Acta. 2013; 1834: 2116-2123.

Baeshen N A, Baeshen M N, Sheikh A, Bora R S, Ahmed M, Ramadan H. Cell factories for insulin production. Microbial Cell Factories. 2014; 13: 141-149.

Boothe J, Nykiforuk C, Shen Y, Zaplachinski S, Szarka S, Kuhlman P, Murray E, Morck D, Moloney MM. Seedbased expression systems for plant molecular farming. Plant Biotechnology Journal. 2010; 8: 588-606.

Boyhan D, Daniell H. Low-cost production of proinsulin in tobacco and lettuce chloroplasts for injectable or oral delivery of functional insulin and C-Peptide. Plant Biotechnology Journal. 2011; 9: 585-598.

Chen LT, Yao M. Characteristic Research and Purification of B23-Gly-B24 human insulin. Pharmaceutieal Bioteellnofogy Bioengineered. 2002; 9: 270-273.

Colagiuri R. Diabetes: a pandemic, a development issue or both? Expert Review of Cardiovascular Therapy. 2010; 8: 305-309.

Ferrer-Miralles N, Domingo-Espín J, Corchero JL, Vázquez E, Villaverde A. Microbial factories for recombinant pharmaceuticals. Microb Cell Fact. 2009; 8: 17-25.

Ferrer-Miralles N, Villaverde A. Bacterial cell factories for recombinant protein production; expanding the catalogue. Microb Cell Fact. 2013; 12: 113.

Gellissen G, Hollenberg C. Application of yeasts in gene expression studies: A comparison of Saccharomyces cerevisiae, Hansenula polymorpha and Kluyveromyces lactis. Gene. 1997; 190: 87-97.

Gurramkonda C, Polez S, Skoko N, Adnan A, Gäbel T, Chugh D. Application of simple fed-batch technique to high-level secretory production of insulin precursor using Pichia pastoris with subsequent purification and conversion to human insulin. Microb Cell Fact. 2010; 9: 31-42.

Hoekema A, Hirsch PR, Hooykaas PJJ, Schilperoort RA. A binary plant vector strategy based on separation of virand T-region of the Agrobacterium tumefaciens Tiplasmid. Nature. 1983; 303: 179-180.

Huang C J, Lin $\mathrm{H}$, Yang $\mathrm{X}$. Industrial production of recombinant therapeutics in Escherichia coli and its recent advancements. J Ind Microbiol Biotechnol. 2012; 39: 383-399.

Jefferson RA, Kavanagh TA, Bevan MW. GUS fusions: beta-glucuronidase as a sensitive and versatile gene fusion marker in higher plants. The EMBO Journal. 1987; 6: 3901.

Kjeldsen T. Yeast secretory expression of insulin precursors. Appl Microbiol Biotechnol. 2000; 54: 277 286.

Markley N, Nykiforuk C, Boothe J, Moloney M. Producing proteins using transgenic oilbody-oleosin technology. Biopharm international. 2006; 19: 34-46.

Martina V, Tagliabue M, Maccario M, D'Antona G, Camanni F. Comparison of Monotard and Ultratard insulin at bedtime in a model of optimized insulin therapy in Italy. Diabete Metab. 1989; 15: 372-374. 
McKeage K, Goa K. Insulin glargine: a review of its therapeutic use as along acting agent for the management of type 1 and 2 diabetes mellitus. Drugs. 2001; 61: 15991624.

Nielsen J. Production of biopharmaceutical proteins by yeast: advances through metabolic engineering. Bioengineered. 2013; 4: 207-211.

Nilsson J, Jonasson P, Samuelsson E, Stahl S, Uhlèn M. Integrated production of human insulin and its Cpeptide. J Biotechnol. 1996; 48: 241-250.

Nykiforuk CL, Boothe JG, Murray EW, Keon RG, Goren HJ, Markley NA, Moloney MM. Transgenic expression and recovery of biologically active recombinant human insulin from Arabidopsis thaliana seeds. Plant Biotechnology Journal. 2006; 4: 77-85.

Pekar AH, Frank B. Conformation of proinsulin. A comparison of insulin and proinsulin self-association at neutral pH. Biochemistry. 1972; 11: 4013-4016.

Peng ZY, Li L, Yang L, Zhang B, Chen G, Bi YP. Overexpression of Peanut Diacylglycerol Acyltransferase 2 in Escherichia coli. PLoS ONE. 2013; 8: e61363.

Peterson G. Intermediate and long-acting insulins: a review of NPH insulin, insulin glargine and insulin detemir. Curr Med Res Opin. 2006; 22: 2613-2619.

Porro D, Sauer M, Branduardi P, Mattanovich D. Recombinant protein production in yeasts. Mol Biotechnol. 2005; 31: 245-259.

Qian X, Kraft J, Ni Y, Zhao F. Production of recombinant human proinsulin in the milk of transgenic mice. Plant Biotechnology Journal. 2011; 9: 585-598.

Ruhlman T, Ahangari R, Devine A, Samsam M, Daniell H. Expression of cholera toxin B-proinsulin fusion protein in lettuce and tobacco chloroplasts-oral administration protects against development of insulitis in non-obese diabetic mice. Plant Biotechnology Journal. 2007; 5: 495-510.

Soltanmohammadi B, Jalali-Javaran M, Rajabi-Memari H, Mohebodini M. Cloning, Transformation and Expression of Proinsulin Gene in Tomato (Lycopersicum esculentum Mill.). Jundishapur J Nat Pharm Prod. 2014; 9: 9-15.
Su L, Zhao CZ, Bi YP, Wan SB, Xia H, Wang X. Isolation and expression analysis of LEA genes in peanut (Arachis hypogaea L.). Journal of Biosciences. 2011; 36: 223228.

Thim L, Hansen MT, Norris K, Hoegh I, Boel E, Forstrom J, Ammerer G, Fiil NP. Secretion and processing of insulin precursors in yeast. PNAS. 1986; 83: 6766-6770.

Trabucchi A, Guerra LL, Faccinetti NI, Iacono RF, Poskus E, Valdez SN. Expression and characterization of human proinsulin fused to thioredoxin in Escherichia coli. Appl Microbiol Biotechnol. 2012; 94: 1565-1576.

Walsh G. Therapeutic insulins and their large-scale manufacture. Appl Microbiol Biotechnol. 2005; 67: 151159.

Walsh G. New biopharmaceuticals. BioPharm Int. 2012; 25: 34-36.

Walsh G. Biopharmaceuticals approval trends in 2013. Biopharm Int. 2013; 26: 54-56.

Wang CT, Huang Y, Yang X, Jang Y, Zhang JC, Chen DX. Isolation of DNA from Peanut: Comparison between Modified CTAB and High Salt, Low $\mathrm{pH}$ Methods. Journal of Peanut Science. 2002; 31: 20-23.

Xia H, Zhao CZ, Hou L, Li AQ, Zhao SZ, Bi YP. Transcriptome profiling of peanut gynophores revealed global reprogramming of gene expression during early pod development in darkness. BMC Genomics. 2013; 14: 517-528.

Xie TT, Qiu QC, Zhang W, Ning TT, Yang W, Zheng CY. A biologically active rhIGF-1 fusion accumulated in transgenic rice seeds can reduce blood glucose in diabetic mice via oral delivery. Peptides. 2008; 29: 18621870 . 